\section{Translation and cultural adaptation of the Multidimensional Scale of Perceived Social Support for Greece}

\section{Paraskevi Theofilou \\ Sotiria Hospital for Thoracic Diseases, Athens, Greece}

\begin{abstract}
Recently, there is a surge of interest in the use of the Multidimensional Scale of Perceived Social Support (MSPSS) to measure perceived social support across cultures. The objective of this study was to translate and make the cultural adaptation of the Greek version of the MSPSS. The study counted with a sample of 10 patients diagnosed with multiple sclerosis. The process involved the following steps of translation back translation and semantic evaluation. The former revealed good acceptance of the translated version of the instrument, which participants considered having items of easy understanding. After completing the process of validation in the country, the instrument will become available to Greek researchers to measure social support, as well as to compare results from Greece to that of other cultures in which the instrument has already been validated.
\end{abstract}

\section{Introduction}

Many studies have addressed the role of social support in predicting the course of chronic disease. Social support is a concept that recognizes patients exist to varying degrees in networks through which they can receive and give aid, and in which they engage in interactions. Social support can be obtained from family, friends, co-workers, spiritual advisors, health professionals, or members of one's community and neighborhood. ${ }^{1-3}$ Social support measurement instruments can facilitate clinical decision making, assess care quality, estimate a population's health service needs and understand the causes and consequences of health problems. ${ }^{4}$ The impact of diseases on the physical health, in the work performance and its implications on the familiar and personal life increase the treatment context. Thus, improvements in the social support become as important as the clinical-laboratorial responses to interventions, enlarging the scope of therapeutic results.
Recently, there is a surge of interest in the use of the Multidimensional Scale of Perceived Social Support (MSPSS) to measure perceived social support across cultures. ${ }^{5-7}$ The MSPSS was initially developed on university students, ${ }^{8}$ then was later validated in a wide range of samples, including psychiatric patients, adolescents, older adults, doctor-trainees and pregnant women. ${ }^{9-11}$

The MSPSS provides assessment of three sources of perceived social support: family (FA), friends (FR), and significant other (S0). Each item is scored on a likert scale ranging from 1 (Very strongly Disagree) to 7 (Very strongly agree) to give a total of score out of 84 , with higher scores indicating greater social support. A cut-off score for service eligibility was set at 65 or less, as it was deemed indicative of sufficiently low levels of perceived social support to warrant intervention. ${ }^{12-14}$ Zimet and his colleagues have argued well the unique features of this scale.5,8 First, it is short (12 items in total) and is ideal for i) research that requires assessment of multiple variables and ii) populations which, for one reason or another, cannot tolerate a long questionnaire. ${ }^{15}$ Second, a point related to ii) above, MSPSS items are easy to understand (requiring just fourth grade reading level) and are therefore suitable for young populations or populations with limited literacy level. Third, despite being a brief instrument, MSPSS measures support from three sources, and in particular, the SO subscale is rather unique among measures in the field. ${ }^{15}$ The MSPSS was found to have good internal reliability across subject groups. In addition, strong factorial validity was demonstrated, confirming the three-subscale structure of the MSPSS: family, friends, and significant other. Finally, strong support was also found for the validity of the family and significant other subscales. ${ }^{11}$ The present research aimed to translate and culturally adapt the MSPSS into the Greek language in a sample of patients diagnosed with multiple sclerosis (MS). MS is a serious chronic disease affecting several aspects of individuals' quality of life, social functioning as well as mental health.18,19 Further, there is little evidence with regards to the social network of these patients in Greece.

\section{Materials and Methods}

This methodological research consists of the translation and cultural adaptation of a social support measurement instrument for chronic disease patients in Greece.

\section{Data collection}

A sample of 10 patients (Table 1) diagnosed
Correspondence: Paraskevi Theofilou, Sotiria Hospital for Thoracic Diseases, Athens, Greece. Tel. $+30.697 .744 .1502,+30.210 .622 .1435$.

Fax +30.210 .622 .1435$

E-mail: theofi@otenet.gr; paraskevi.theofilou@ gmail.com

Key words: translation, cultural adaptation, social support, multiple sclerosis.

Conflict of interests: the author declares no potential conflict of interests.

Received for publication: 9 February 2013.

Accepted for publication: 2 March 2013.

This work is licensed under a Creative Commons Attribution NonCommercial 3.0 License (CC BYNC 3.0).

(C) Copyright P. Theofilou, 2015

Licensee PAGEPress, Italy

Health Psychology Research 2015; 3:1061

doi:10.4081/hpr.2015.1061

with MS was recruited from a General Hospital in the broader area of Athens. Selection criteria included: i) >18 years of age; ii) ability of communication in Greek; iii) diagnosed with MS; iv) satisfying level of cooperation and perceived ability

The rate of response was very high, reaching $100 \%$. Thus, the total sample includes all patients with a mean age of $50.1 \pm 8.47$ years. Participants were Greek adults having signed a consent form for participation. All subjects had been informed of their rights to refuse or discontinue participation in the study according to the ethical standards of the Helsinki Declaration. Ethical permission for the study was obtained from the scientific committee of the participating hospital.

\section{Translation and back translation of the Multidimensional Scale} of Perceived Social Support

The MSPSS version was translated from the source language (English) to the target language (Greek). Translation was done according to the guidelines for adapting instruments in multiple languages and cultures. ${ }^{16}$ Translators who were conversant with both the source and target languages, and had skills in cross-cultural adaptation of instruments, made two independent forward translations and two independent backward translations. The final version was independently reviewed and translated by a bilingual health psychologist without previously seeing the original MSPSS. The back translated version had very close concordance with the original MSPSS, as verified by a professional linguist fluent in both the English and Greek languages. A social scientist 
conversant with both languages carried out the final step of smoothing out the language. This involved editing the target language version of the instrument in a consistent writing style. This helped to ensure that patients could easily understand the modified version of the MSPSS. A social support expert reviewed the final instrument to check for omissions (see Appendix for the final version, in Greek).

\section{Semantic validation of the \\ Multidimensional Scale of Perceived Social Support}

Next, semantic validation was carried out, which serves to verify the understanding of existing MSPSS items by interviewing the respondents. This phase aimed to identify problems related to the research subjects' understanding and acceptance of the terms. For this phase of the cultural adaptation process, all 10 MS patients answered the MSPSS as well as the General Impression Instrument.

\section{Results}

As mentioned, 10 MS patients participated in the semantic validation phase, without any refusals. The goal of this study phase was to identify possible problems to understand the instrument's items and answer categories, with a view to adjusting terms for adaptation to the Greek culture if necessary. Therefore, an interview was held, in which patients, who agreed to participate in the study after receiving information, signed two copies of the Informed Consent Term (ICT) and answered the above mentioned forms. The analysis of answers to the General Impression instrument revealed that, in general, the participants accepted well the MSPSS and found it easy to understand. ${ }^{17}$ In total, $90.0 \%$ of the patients considered the instrument very good, and items were found relevant, easy to understand and with appropriate alternative answer categories for the chronic condition under analysis. The results are displayed in Table 2. With regards to the assessment of social support, MS patients presented lower scores in the scale of significant others $(15.20 \pm 3.67)$. The scores indicated in the scales of family and friends were $23.20 \pm 3.67$ and $17.00 \pm 2.11$ respectively. The total score was $55.40 \pm 8.83$.

\section{Discussion and Conclusions}

MS is a neurodegenerative disease characterized by chronic inflammation, demyelination, and scarring of the central nervous system. Symptoms include weakness, fatigue, sensory loss, vertigo, lack of coordination, impotence or sexual dysfunction, urinary incontinence, optic atrophy, dysarthria, and mental problems. ${ }^{18,19}$

MS has a major impact on the lives of patients. The disease substantially interferes with daily activities and family, social and working life, disturbs emotional well-being, and reduces quality of life. ${ }^{18,19}$ In our study, MS patients present low levels of perceived social support according to the cut-of-points of the MSPSS. This finding is in agreement with other studies' results showing the chronic disease patients' negative evaluation of their social network. 20

There are few social support measurement instruments and, today, there is no specific instrument for use in Greece. Therefore, in this study, internationally adopted procedures were followed for the cultural adaptation and validation of instruments to measure subjective constructs.

In the cultural adaptation and validation process of the MSPSS, in the semantic part, MS patients self-applied the instrument, followed by an interview, during which the understanding and acceptance of the terms deriving from the translation process were verified. As a result, $90.0 \%$ of them considered the items easy to understand and the answer categories adequate, providing answers without any difficulty. This study permitted multidisciplinary contact, involving statisticians, physicians, nurses and psychologists, which was fundamental for the cultural adaptation process of an instrument that serves to measure a subjective health-related construct. As this instrument is directed at chronic patients as well, different professionals' involvement permitted greater knowledge on the several aspects involved. Every day, health professionals interact with patients who go through situations of anxiety and discomfort due to chronic conditions. The responsibility to relieve symptoms demands the assessment of physical, physiological, emotional, behavioral and environmental aspects that influence the patient's condition. Thus, in clinical medicine, social support assessment instruments need to be used as indicators to direct actions with a view to comprehensive care delivery.

A very important limitation of the present paper relates to the fact that the sample selected is very small due to the aim of the study which was the translation and cultural adaptation of the questionnaire. The next step is to validate the specific instrument using larger populations and assessing in this way its psychometric properties.
Table 1. Characteristics of the sample $(\mathrm{n}=10)$.

\begin{tabular}{lc} 
Age, mean \pm SD & $50.1 \pm 8.47$ \\
Gender & \\
Male & 4 \\
Female & 6 \\
Marital status & \\
Single & 2 \\
Married & 7 \\
Divorced & 1 \\
$\quad$ Widowed & 0 \\
\hline Education & \\
$\quad$ Elementary & 1 \\
$\quad$ Secondary & 6 \\
$\quad$ University & 3 \\
Length of the treatment in years, & $9.40 \pm 4.22$ \\
mean \pm SD & \\
\hline SD, standard deviation.
\end{tabular}

SD, standard deviation.
Table 2. Assessment results for the General Impression part of the semantic validation phase of the Multidimensional Scale of Perceived Social Support instrument.

\begin{tabular}{llc}
\hline $\begin{array}{l}\text { Items from the general } \\
\text { impression instrument }\end{array}$ & Alternative answers & Answers, \% \\
What did you think about our & & \\
questionnaire in general? & Very good & 90 \\
Are questions understandable? & Good & 10 \\
& Easy to understand & 90 \\
\hline About the answer categories: & Sometimes difficult & 10 \\
did you have any difficulties? & No difficulty & 100 \\
Are the questions important & Some difficulties & 0 \\
for your health condition? & Very relevant & 90 \\
\hline
\end{tabular}




\section{References}

1. Patel SS, Peterson RA, Kimmel PL. The impact of social support on end-stage renal disease. Semin Dial 2005;18:98-102.

2. House JS, Landis KR, Umberson D. Social relationships and health. Science 1988;241: 540-5.

3. Theofilou P. The relation of social support to mental health and locus of control in chronic kidney disease. J Renal Nurs 2012;4:18-22.

4. Theofilou P. Outcome measurement in palliative care: quality of life. J AIDS Clin Res 2012;3:1-6.

5. Canty-Mitchell J, Zimet GD. Psychometric properties of the multidimensional scale of perceived social support in urban adolescents. Am J Commun Psychol 2000;28:3 91-400.

6. Chou K.L. Assessing Chinese adolescents, social support: the multidimensional scale of perceived social support. Pers Individ Dif 2000;28;299-307.

7. Eker D, Arkar H, Yaldiz H. Generality of support sources and psychometric properties of a scale of perceived social support in Turkey. Soc Psychiatry Psychiatr
Epidemiol 2000;35:228-33.

8. Zimet GD, Dahlem NW, Zimet SG, Farley GK. The multidimensional scale of perceived social support. J Pers Assess 1988; 52:30-41.

9. Kazarian SS, McCabe SB. Dimensions of social support in the MSPSS: factorial structure, reliability, and theoretical implications. J Commun Psychol 1991;19:15060 .

10. Stanley MA, Beck JG, Zebb BJ. Psychometric properties of the MSPSS in older adults. Aging Mental Health 1998;2:186-93.

11. Zimet GD, Powell SS, Farley GK, et al. Psychometric properties of the multidimensional scale of perceived social support. J Pers Assess 1990;55:610-7.

12. Gallegos D. Managing work and motherhood: implications for perinatal mental health. Perth: State Perinatal Reference Group, Department of Health, CSCR and WACRW; 2007.

13. Husain N, Bevc I, Husain MI, et al. Prevalence and social correlates of postnatal depression in a low income country. Arch Mental Health 2006;9:197-202.

14. Roman L, Lindsay J, Moore JS, et al. Addressing mental health and stress in medicaid-insured pregnant women using a nurse-community health worker home visiting team. Publ Health Nurs 2007;24:239-48.

15. Cheng ST, Chan ACM. The multidimensional scale of perceived social support: dimensionality and age and gender differences in adolescents. Pers Individ Dif 2004;37:1359-69.

16. Hambleton RK. Issues, designs and technical guidelines for adapting tests in multiple languages and cultures. In: Hambleton RK, Merenda PF, Spielberg CD. Adapting educational and psychological tests for cross-cultural assessment. Mahwah: Lawrence Erlbaum Associates; 2002.

17. Theofilou P, Kapsalis F, Panagiotaki H. Greek version of MVQOLI-15: translation and cultural adaptation. Int $\mathrm{J}$ Caring Sci 2012;5:289:94.

18. Theofilou P. Quality of life, depression and fatigue in multiple sclerosis patients. $\mathrm{J}$ Neurol Neurophysiol 2011;2:1-2.

19. Miller A, Dishon S. Health-related quality of life in multiple sclerosis: the impact of disability, gender and employment status. Qual Life Res 2006;15:259-71.

20. Chan LK, Yu ECS, Li SY. Depression in patients receiving peritoneal dialysis. East Asian Arch Psychiatry 2011;21:99-107. 